\title{
APPLICATION OF FAST IMPLODING CAPILLARY DISCHARGE FOR LASER WAKEFIELD ACCELERATION
}

\author{
$\underline{\text { T. Hosokai* }}^{*}$ M. Kando, H. Dewa, H. Kotaki, S. Kondo, N. Hasegawa \\ Advanced Photon Research Center, Kansai Research Establishment \\ Japan Atomic Energy Research Institute, Tokai-mura Naka-gun Ibaraki-ken 319-1195 Japan \\ K. Horioka, M. Nakajima, Department of Energy Sciences, Tokyo Institute of Technology \\ Nagatsuta 4259, Midori-ku Yokohama 226-8502 Japan \\ K. Nakajima, High Energy Accelerator Research Organization (KEK) \\ 1-1 Oho Tsukuba-shi Ibaraki-ken 305-0801 Japan
}

\begin{abstract}
A new method of optical guiding for the laser wakefield acceleration using an imploding phase of a gas-filled fast capillary discharge has been proposed. An imploding plasma column has a concave electron density profile in the radial direction just before a stagnation phase driven by a converging current sheet and shock wave. The feasibility of optical guiding of high intensity laser pulses using an imploding phase of the fast capillary discharge has been experimentally demonstrated over a distance of $2 \mathrm{~cm}$ corresponding to $\sim 12.5$ times the Rayleigh length. A high intensity laser pulse $\left(>10^{17} \mathrm{~W} / \mathrm{cm}^{2}\right)$ focused on the front edge of the capillary has propagated through the center of the column maintaining its spot size. The electron density in the fully ionized channel is estimated to be $6.0 \times 10^{16} \mathrm{~cm}^{-3}$ on the axis and $1.5 \times 10^{18} \mathrm{~cm}^{-3}$ on the peaks with the diameter of $70 \mu \mathrm{m}$.
\end{abstract}

\section{INTRODUCTION}

In order to increase the energy gain of electrons accelerated by the laser wake-fields, it is a critical issue to propagate a high intensity short laser pulse in a plasma larger than the vacuum Rayleigh length limited by diffraction. [1] Several methods for extending the propagation distances of intense laser pulses have been proposed: relativistic self-guiding in a plasma [2] and guiding in preformed plasma channels generated by a focused laser pulse [3] or by slow discharge through a capillary in vacuum.[4] For the optical guiding of laser pulses, the electron density profile must be symmetric in the radial direction and have a minimum on the axis, causing the wavefront to curve inward and the laser beam to converge. When this focusing force is strong enough to counteract the diffraction of the beam, the laser pulse can propagate over a long distance and maintain a small beam spot size in a plasma. For the guiding of intense ultrashort laser pulses shorter than the plasma wavelength, it is predicted that the relativistic self-channeling is ineffective in preventing diffraction, but the preformed plasma channel

\footnotetext{
*Email:thosokai@hikari.tokai.jaeri.go.jp
}

can provide a robust optical guiding.[5]

We have presented the first direct observation of optical guiding of high intensity laser pulses over $2 \mathrm{~cm}$ through a plasma channel produced by an imploding phase of fast Z-pinch discharge in a gas-filled capillary.

\section{FAST CAPILLARY DISCHARGE}

A high current fast Z-pinch discharge generates strong azimuthal magnetic field, which contracts the plasma radially inward down to $\sim 100 \mu \mathrm{m}$ in diameter. The imploding current sheet drives the converging shock wave ahead of it, producing a concave electron density profile in the radial direction just before the stagnation phase. The concave profile is approximately parabolic out to a radius of $\sim 50$ $\mu \mathrm{m}$, after which the density falls off.

In the research of capillary discharge pumped X-ray laser, it has been shown that stable and reproducible channel can be produced by this scheme, and this scheme can be scalable to form longer and higher density channels by tailoring of the implosion. [6] It was reported that a stable, liner plasma column over the length of $12 \mathrm{~cm}$ could be produced at an electron density of more than $10^{19} \mathrm{~cm}^{-3}$. [7]

For the laser wakefield acceleration (LWFA), a plasma channel with a density in the order of $10^{17} \mathrm{~cm}^{-3}$ and a length of $\sim 10 \mathrm{~cm}$ will be required. [1] We have started a study of optical wave-guiding in the density range of $10^{16}$ $-10^{18} \mathrm{~cm}^{-3}$ as the first step for application of the channel guided LWFA. Since the density profile in the capillary depends strongly on the discharge process, we have experimentally investigated the discharge dynamics and plasma channel formation.

\section{OPTICAL GUIDING EXPERIMENT}

The typical experimental setup is shown in Fig 1. We have used a capillary with an inner diameter of $1 \mathrm{~mm}$ and a length of up to $2 \mathrm{~cm}$ which was bored at the central axis of an alumina $\left(\mathrm{Al}_{2} \mathrm{O}_{3}\right)$ rod of $50 \mathrm{~mm}$ in diameter. The capillary load was placed between two electrodes with an in- 
ner diameter of $400 \mu \mathrm{m}$ which were made of molybdenum. The electrodes were cylindrically connected to a thyratron (EG\&G HY-5) and four ceramic capacitors of $2 \mathrm{nF}$ using four coaxial cables. The capacitor was charged up to 20 $\mathrm{kV}(1.6 \mathrm{~J})$. With this configuration, the discharge current which was monitored by a Rogowski coil having a peak of $2.4 \mathrm{kA}$ with a rise time of about $20 \mathrm{~ns}$ and a duration of 70 ns (FWMH). The capillary was filled with helium, under differential pumping at an initial pressure which was varied from 0.5 to 5 Torr. A DC discharge circuit was used to form an uniformly preionized helium gas.

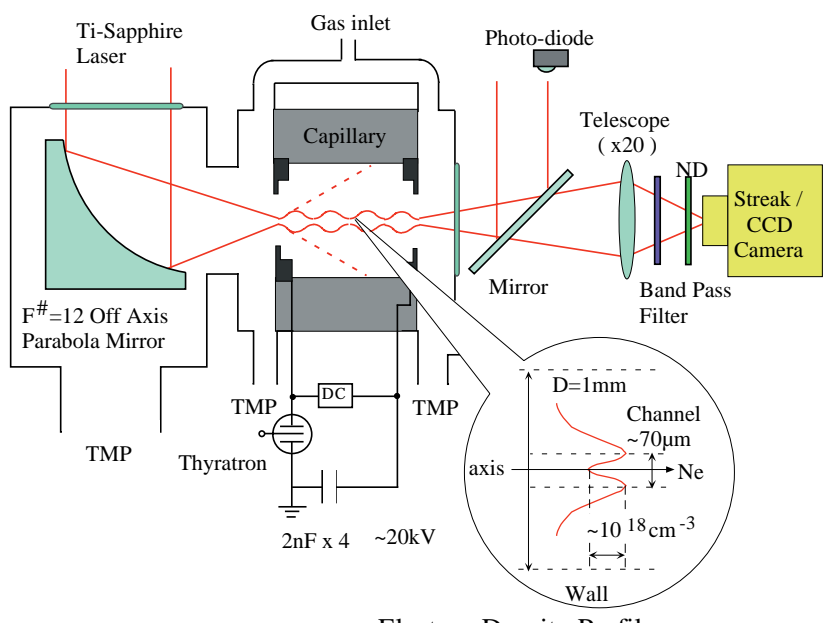

Electron Density Profile

Figure 1: Experimental setup of a fast capillary discharge for a plasma waveguide. A typical electron density profile of the implosion phase of the fast capillary discharge in the radial direction is illustrated in the circle.

The discharge dynamics was observed with a streak camera (HAMAMATSU C-2830) placed on the capillary axis. The visible light emission from the capillary was imaged on the streak-camera slit through a telescope $(\times 20)$. To investigate the guiding channel formation in the capillary, a He-Ne laser beam $(\lambda=632.8 \mathrm{~nm})$ was focused on the front edge of the capillary to a spot size of $40 \mu \mathrm{m}$ in diameter by means of a $F^{\#}=20$ lens. The transmitted He-Ne laser beam profile at the exit of the capillary was observed through a band pass filter $(\Delta \lambda=1 \mathrm{~nm})$, with the streak camera. As a further investigation, a high intensity Ti-Sapphire laser pulse $\left(\lambda=790 \mathrm{~nm}, 90 \mathrm{fs},>1 \times 10^{17} \mathrm{~W} / \mathrm{cm}^{2}\right)$ was focused on the front edge of the capillary to a spot size of 40 $\mu \mathrm{m}$ in diameter by means of a $F^{\#}=12$ off-axis parabolic mirror. The transmitted laser beam profile at the exit of the capillary was observed through a band pass filter $(\Delta \lambda=10$ $\mathrm{nm})$, with a CCD camera.

\section{RESULTS}

Figure. 2 shows a typical streak image of the plasma emission for the initial pressure of 0.9 Torr, together with the intensity profile along the radial direction at $\mathrm{t}=8.5 \mathrm{~ns}$. The emission from only the central portion of the capil- lary with a diameter less than $400 \mu \mathrm{m}$ was imaged on the streak camera. We found that the compression of the column produced a luminous region at $\mathrm{t}=2.5 \mathrm{~ns}$, which exhibits good symmetry with respect to the axis. Then, the luminous region on the axis faded out during expansion of the column. Later, the second implosion driven by a larger current produced a brighter luminous region at $\mathrm{t}=8.5 \mathrm{~ns}$. It again maintains good symmetry with respect to the axis. In addition, as shown in Fig.2(b), it had a clear dip with a width of $70 \mu \mathrm{m}$ on the axis. The column oscillated in the radial direction several times while the main power pulse was alive.
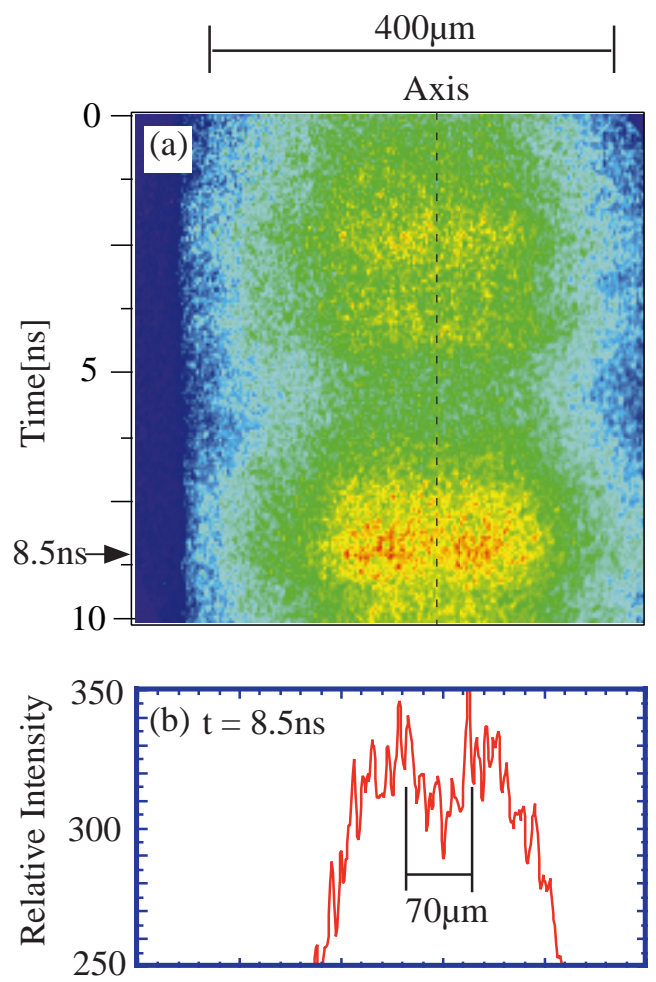

Figure 2: (a) A typical streak image of the capillary discharge at an initial pressure of 0.9 Torr He : $t=0$ corresponds to the beginning of the main discharge pulse. (b) The radial intensity profile of the plasma emission was $\mathrm{t}=$ $8.5 \mathrm{~ns}$.

We have observed the brightness and the radial profile of the transmitted He-Ne laser beam and high intensity Tisapphire laser pulses through the capillary at the exit. A typical streak image of the transmitted He-Ne laser beam profile through the capillary discharge plasma can be seen in Fig.3. Since the observed time window of Fig.3 almost coincided with that of Fig.2(a), the beam profile evolution could be correlated with the discharge dynamics. As shown in Fig.3, the beam radius gradually contracted in the second implosion phase and a brightly enhanced smaller beam spot could be observed at the time $(\mathrm{t} \sim 8.5 \mathrm{~ns}$ ) when the luminous profile exhibited double peaks in Fig.2. The observed spot image size ( diameter ) was $\sim 40 \mu \mathrm{m}$ at the exit of the capillary. These results suggest that the guiding 
channel was uniformly formed in the core of the column at the second implosion phase. Figure 4 shows typical CCD images of the transmitted high intensity Ti-sapphire laser pulse profile through the capillary discharge plasma at $\mathrm{t}=$ $0 \mathrm{~ns}$ (a) and at $\mathrm{t} \sim 8.5 \mathrm{~ns}$ (b). These show clearly that a high intensity laser pulse could be guided through the channel over a distance of $2 \mathrm{~cm}$ corresponding to $\sim 12.5$ $Z_{R 0}$, where $Z_{R 0} \sim 1.6 \mathrm{~mm}$ is the Rayleigh length of the Ti-Sapphire laser system.

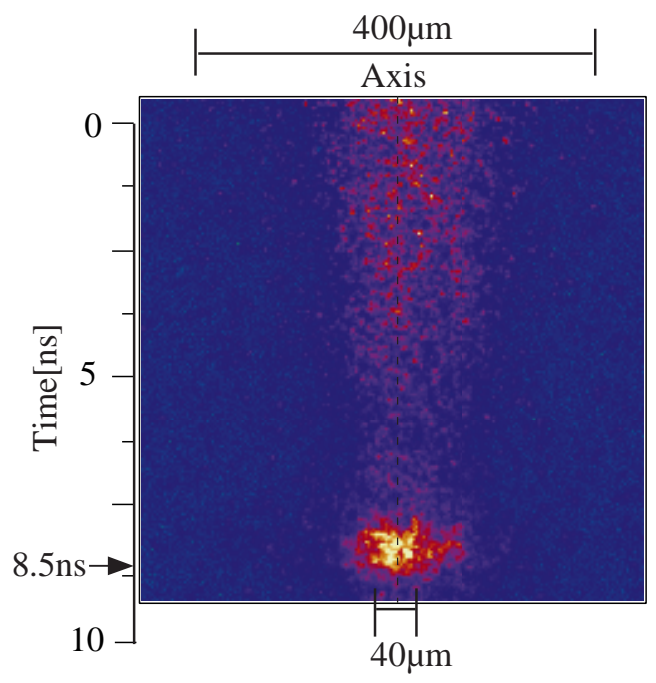

Figure 3: A typical streak image of the transmitted He-Ne laser beam through the capillary at an initial pressure of 0.9 Torr He : $t=0$ corresponds to the beginning of the main discharge pulse.

The electron density profile in the channel was estimated by corroborating the transmitted laser images with the results of ray trace calculation [8]. We assumed that the electron density profile of the channel in the radial direction was parabolic and the channel was fully ionized with the electron density on the axis is of $6.0 \times 10^{16} \mathrm{~cm}^{-3}$ which was twice the initial gas density. These were based on results of the 1D-MHD simulation using code MULTI-Z. $[6,9,10]$ We also assumed that the incident laser had a radial Gaussian profile, the channel was uniform over the channel length and the channel radius $r_{c h}$ was $35 \mu \mathrm{m}$ according to the observed value. Under these assumptions, we can estimate the electron density on the peaks of the channel to be $1.5 \times 10^{18} \mathrm{~cm}^{-3}$ and the density gradient to be $\sim 4 \times 10^{20} \mathrm{~cm}^{-4}$. The matched beam radius $r_{m}$ was given by $r_{m}=\left[r_{c h}^{2} /\left(\pi r_{e} \Delta n\right)\right]^{1 / 4}$, where $\Delta n$ was the channel depth and $r_{e}=e^{2} / m c^{2}$ was the classical electron radius. [5] With $r_{c h}=35 \mu \mathrm{m}$ and $\Delta n \sim 1.5 \times 10^{18} \mathrm{~cm}^{-3}$, the matched beam radius is $r_{m} \sim 17 \mu \mathrm{m}$. The observed spot radius of $\sim 20 \mu \mathrm{m}$ was consistent with this value.

\section{SUMMARY}

In summary, we have presented the optical guiding of high intensity laser pulse using an imploding phase of a gas-
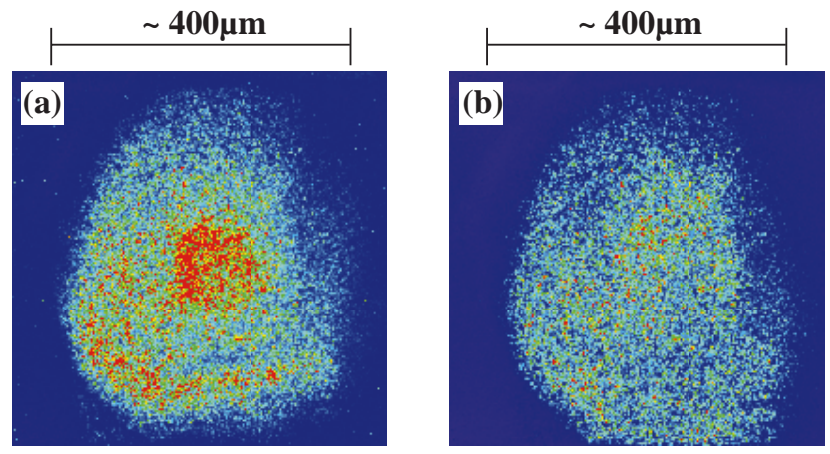

Figure 4: Typical CCD images of the transmitted a high intensity Ti-Sapphire laser pulse $\left(\sim 1 \times 10^{17} \mathrm{~W} / \mathrm{cm}^{2}\right)$ through the capillary at an initial pressure of 0.9 Torr He: The capillary length $\mathrm{L}=2 \mathrm{~cm}$. (a) $\mathrm{t}=0 \mathrm{~ns}$ (b) $\mathrm{t} \sim 8.5 \mathrm{~ns}$.

filled capillary discharge and experimentally investigated the guiding channel formation in the capillary. The results indicate that the imploding column could uniformly produce a concave electron density profile in its core over a distance of $2 \mathrm{~cm}$. The electron density in the fully ionized channel was estimated to be $6.0 \times 10^{16} \mathrm{~cm}^{-3}$ on the axis and $1.5 \times 10^{18} \mathrm{~cm}^{-3}$ on the peaks with a diameter of 70 $\mu \mathrm{m}$. The optical guiding of high intensity laser pulses $(>$ $1 \times 10^{17} \mathrm{~W} / \mathrm{cm}^{2}$ ) was experimentally demonstrated in the second imploding phase of the discharge.

\section{REFERENCES}

[1] K. Nakajima, Phys. Plasmas. 3, 2169 (1996)

[2] K. Krushelnick, A. Ting, C. I. Moore, H. R. Burris, E. Esarey, P. Sprangle, and M. Baine, Phys. Rev. Lett. 78, 4047 (1997)

[3] C. G. Durfee III and H. M. Milchberg, Phys. Rev. Lett. 71, 2409 (1993)

[4] Y. Ehrlich, C. Cohen, A. Zigler, J. Krall, P. Sprangle, and E. Esarey, Phys. Rev. Lett. 77, 4186 (1996)

[5] P. Sprangle, E. Esarey, J. Krall, and G. Joyce, Phys. Rev. Lett. 69, 2200 (1992)

[6] ] T. Hosokai, M. Nakajima, T. Aoki, M. Ogawa, and K. Horioka, Jpn. J. Appl. Phys. 36, 2327 (1997)

[7] J. J. Rocca, V. N. Shlyaptsev, F. G. Tomasel, O. D. Cortazar, D. Hartshorn, and J. L. A. Chilla, Phys. Rev. Lett. 73, 2192 (1994)

[8] H. Hanajima, M. Nakajima, and K. Horioka, Proc. 6th International Conference on X-ray lasers, Kyoto Japan 1998, to be published

[9] R. Ramis, R. Schmalz, and J. Meyer-Ter-Vehen, Comput. Phys. Commun. 49, 475 (1988)

[10] T. Aoki, K. Horioka, and M. Ogawa, NIFS-Proc-26 Res. Rep. Natl. Inst. Fusion Sci., 179 (1996) 